\title{
Endovascular Therapy of Hypoglossal Canal AVFs Presenting with Orbital Symptoms
}

\author{
Vladislav Miropolsky, Leodante B. da Costa, Thomas R. Marotta, Julian Spears
}

\begin{abstract}
Objective: Dural arteriovenous fistulae (DAVF) of the hypoglossal canal region are rare lesions. We describe three cases of DAVF of the hypoglossal canal presenting with ocular symptoms and discuss the endovascular management options. Methods: Three consecutive patients with DAVF of the hypoglossal canal region presented with proptosis, chemosis and disturbances of extra-ocular mobility. Each patient was treated using a different endovascular approach, based on variations of the vascular access. Results: The cases and treatments are reviewed, with a literature review on the subject. Endovascular treatment, transvenous or trans-arterial was curative in all cases. Conclusion: DAVF of the hypoglossal canal region can present with ocular manifestations very similar to DAVF of the cavernous sinus or carotid-cavernous fistulas. Endovascular treatment is usually feasible and effective, but an understanding of the vascular anatomy and pathophysiology of the disease are of utmost importance when planning the approach.
\end{abstract}

RÉSUMÉ: Traitement endovasculaire des fistules artérioveineuses du canal de l'hypoglosse avec symptômes orbitaires. Objectif : Les fistules artérioveineuses durales (FAVD) de la région du canal de l'hypoglosse sont rares. Nous décrivons trois cas de FAVD du canal de l'hypoglosse dont les symptômes initiaux étaient des symptômes oculaires et nous discutons des options endovasculaires de traitement. Méthodes : Trois patients consécutifs atteints de FAVD de la région du canal de l'hypoglosse ont consulté pour exophtalmie, chémosis et troubles de la motricité extra-oculaire. Chaque patient a été traité au moyen d'une approche endovasculaire différente à cause de variations individuelles de l'accès vasculaire. Résultats : Les cas et les traitements ont été révisés et nous avons procédé à une revue de littérature sur ce sujet. Le traitement endovasculaire, transveineux ou transartériel, s'est avéré curatif dans tous les cas. Conclusion : Les FAVD de la région du canal de l'hypoglosse peuvent provoquer des manifestations oculaires comme symptômes initiaux qui sont très semblables à celles des FAVD du sinus caverneux ou des fistules carotidiennes-caverneuses. Le traitement endovasculaire est habituellement faisable et efficace, mais une compréhension de l'anatomie vasculaire et de la physiopathologie de la maladie sont cruciales pour déterminer l'approche à privilégier.

Can. J. Neurol. Sci. 2009; 36: 745-750

Dural arteriovenous fistulae (DAVF) are abnormal arteriovenous shunts that occur in the dura. Clinical manifestation is related to the pattern of venous drainage and can include ocular symptoms, bruit, congestive venous encephalopathy and intracranial hemorrhage. Approximately $50 \%$ of dural arteriovenous fistulae occur in the posterior fossa, and typically involve the transverse and sigmoid sinuses. Involvement of the tentorium or marginal sinus is less common. Borden et al have classified cranial DAVF as follows: Type I DAVFs are those that drain directly into the dural venous sinus or meningeal vein. Type II DAVFs are those that drain into the venous sinus with retrograde drainage into subarachnoid (superficial) veins. Type III DAVFs are those that drain into the subarachnoid veins. Type I DAVFs usually present with minor symptoms. In contrast, Types II and III DAVFs cause serious problems and require treatment. ${ }^{1}$

From the Division of Neurosurgery (JS), Diagnostic \& Therapeutic Neuroradiology (TRM), St. Michael's Hospital, Department of Medical Imaging (VM), University of Toronto; Division of Neurosurgery (LBdC), Sunnybrook Hospital, Toronto, Ontario. Received March 26, 2009. Final Revisions Submitted June 16, 2009.

Correspondence to: Julian Spears, Division of Neurosurgery, St. Michael's Hospital, University of Toronto, Suite 1005, 2 Queen Street East, Toronto, Ontario, M5C 3G7, Canada. 

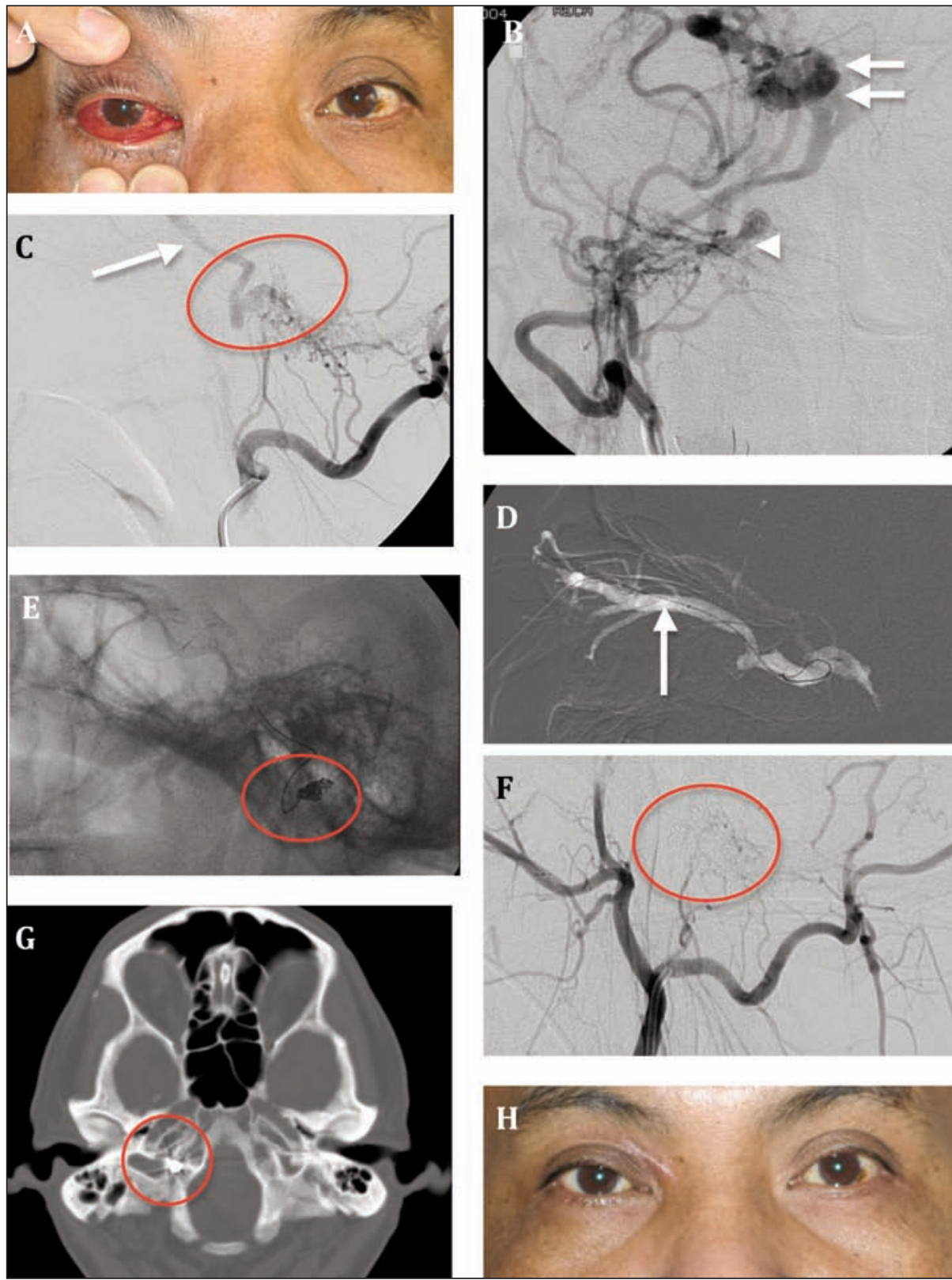

Figure 1: (A) Patient presented with right eye chemosis. (B) and (C) Right external carotid artery injection shows DAVF in the hypoglossal canal (within red border) supplied by multiple branches of the occipital and ascending pharyngeal arteries; venous drainage occurred through right IPS (arrowhead), cavernous sinus (double arrows), superior ophthalmic vein (white arrow) and facial vein. (D) Cannulation of right superior ophthalmic vein (white arrow) for access to venous pouch. (E) embolization of the fistulous pouch with multiple coils. $(F)$ External carotid artery injection shows complete occlusion of the fistula (within red border). $(G)$ Embolization coils deployed in intraosseous hypoglossal canal DAVF. (H) Resolution of occular findings at follow-up.

Dural arteriovenous fistulae located in the hypoglossal canal region have been described but are extremely rare. Patients may present with cranial nerve neuropathy or symptoms related to increased intracranial or intraocular venous pressure. The relative distance and apparent disconnection between the eyes and the hypoglossal canal may be misleading when investigating patients with ocular symptoms secondary to increased intraocular venous pressure. The treatment of these lesions can be challenging due to the complex anatomy with highly varied arterial supply and venous drainage patterns.

We present three cases of dural arteriovenous fistulae located in the hypoglossal canal region, all presenting with orbital symptoms. Each of those lesions was managed with a different endovascular approach, and a discussion of the treatment options is provided. 


\section{Chinical Cases}

\section{Case 1: Superficial Venous Approach (Superior Ophthalmic Vein)}

A 50-year-old male with a history of a long-standing audible bruit on the right presented with an abrupt onset of right eye chemosis, proptosis and glaucoma. Physical examination revealed right 6 th nerve palsy and a chemotic, proptotic eye with increased intraocular pressure. Visual acuity was stable. Magnetic resonance imaging of the brain revealed edema and diffuse thickening of extra-ocular eye muscles and a dilated right superior ophthalmic vein. A CT venogram showed no connection between the right internal jugular vein and ipsilateral inferior petrosal sinus (IPS). The IPS communicated, however, with an intraosseous venous channel traversing the right hypoglossal canal. Digital subtraction angiography showed a dural AVF of the right hypoglossal canal supplied by the neuromeningeal branch of the right ascending pharyngeal artery and multiple small arteries originating from the occipital artery. Retrograde venous drainage of the fistula occurred through the IPS to the cavernous sinus and through the superior ophthalmic vein and facial vein with cortical venous reflux. A superior ophthalmic vein approach was selected after an unsuccessful attempt of an approach through the right internal jugular vein. The right superior ophthalmic vein was dissected and cannulated using a direct surgical cut-down. A total of six fibered GDC platinum coils (Boston Scientific Target) were placed in the fistulous pouch of the right hypoglossal canal with complete occlusion of the fistula and immediate improvement in the patient's symptoms (Figure 1).

\section{Case 2: Jugular Venous Approach (Jugular Vein)}

A 74-year-old male presented with an eight-month history of persistent right eye chemosis. Cerebral angiography showed a DAVF of the right hypoglossal canal region supplied

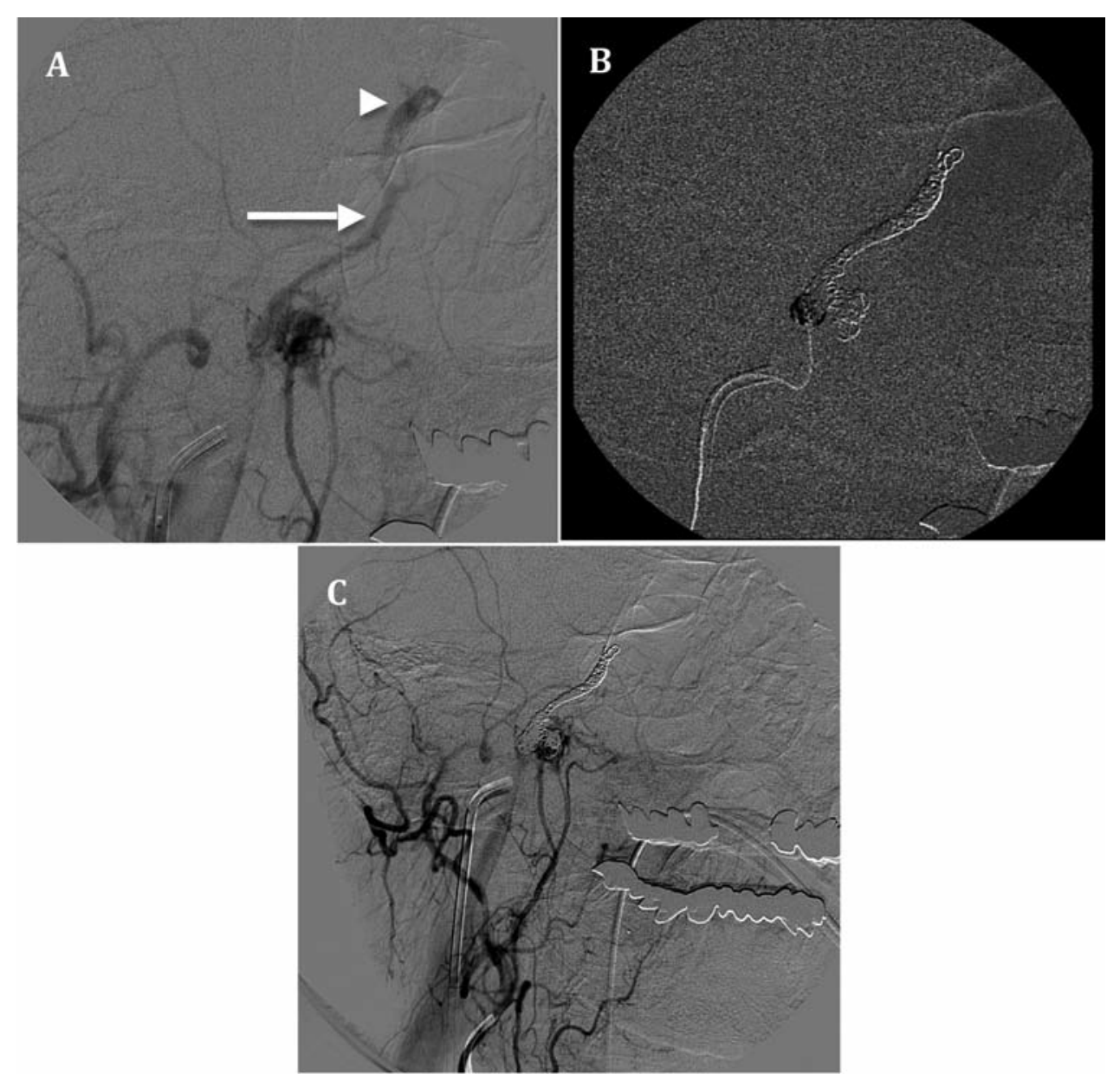

Figure 2: (A) Arterial injection demonstrates a DAVF in the hypoglossal canal with main arterial supply from right ascending pharyngeal artery and stylomastoid branch of the occipital artery. Venous drainage is up through IPS (arrow) and through the cavernous sinus (arrowhead) into the orbital venous system. (B) Jugular venous access for deposition of multiple coils from mid portion of the IPS towards the venous pouch within the hypoglossal canal. (C) Post procedural angiogram demonstrates coils filling the venous pouch. The superior ophthalmic vein retrograde drainage has now been excluded. 
predominantly by the neuromeningeal branch of the right ascending pharyngeal artery and a stylomastoid branch of the occipital artery. Venous drainage of the fistula was in both an anterograde direction through the jugular bulb and jugular vein as well as in a retrograde direction via the IPS, cavernous sinus and superior ophthalmic vein. Superselective angiography was performed and through a transvenous approach via the right jugular vein, seventeen bare and fibered coils were placed into the mid-portion of the IPS coiling in a caudal direction towards the venous pouch located in the hypoglossal canal region, disconnecting completely the retrograde drainage towards the eye. The patient's symptoms improved immediately with complete resolution at follow up in six weeks (Figure 2).

\section{Case 3: Transarterial Approach}

A 50-year-old female presented with right chemosis, diplopia and headache. Computed tomograhy suggested the presence of a fistula and a cerebral angiogram confirmed a Borden type III DAVF involving the region of the hypoglossal canal supplied by multiple branches of bilateral external carotid arteries and vertebral arteries, and the cavernous segment of the right internal carotid artery. Venous drainage was primarily via the right IPS, with reflux into right sphenoparietal sinus, right superior ophthalmic vein and cortical veins. A venous approach through both internal jugular veins and the left IPS (an attempt to cross transclival or transsellar venous anastomoses into the right IPS) was unsuccessful. Transarterial embolization initially with polyvinyl alcohol to reduce flow through the left stylomastoid and left ascending pharyngeal arteries and eventually with nbutyl-cyanoacrylate using superselective catheterization of right ascending pharyngeal artery was done. Venous penetration of embolic material into the right IPS and right cavernous sinus was noted on the final angiogram on the day of treatment and a follow-up cerebral angiogram two weeks later confirmed complete occlusion of the DAVF. The patient's symptoms resolved completely (Figure 3 ).

\section{Discussion}

Dural arteriovenous fistulae are uncommon lesions. Clinical presentation can vary widely according to the pattern of venous drainage, ranging from benign symptoms that include bruit or orbital/ocular symptoms to more ominous presentation and natural history, with a high rate of intracranial hemorrhage, when cortical venous reflux is present. ${ }^{2-5}$ Although the pathogenesis of DAVFs is not yet well understood, they are considered acquired lesions, and local angiogenic factors, hypoxia and trauma may play an important role in their development. ${ }^{6-13}$ Despite the fact that approximately $50 \%$ of the lesions are located in the posterior fossa, involvement of the hypoglossal canal region is extremely rare.

Normal MR imaging appearance of the hypoglossal canal includes variable size of the canal that contains a combination of venous enhancement and occasional linear non-enhancement of the hypoglossal nerve rootlets. Intracranial extension of enhancement beyond the confines of the bony canal is also recognized. Stuckey has described a case of marked asymmetric protrusion of the hypoglossal canal emissary veins into the cerebellomedullary cisterns with potential to mimic diseases
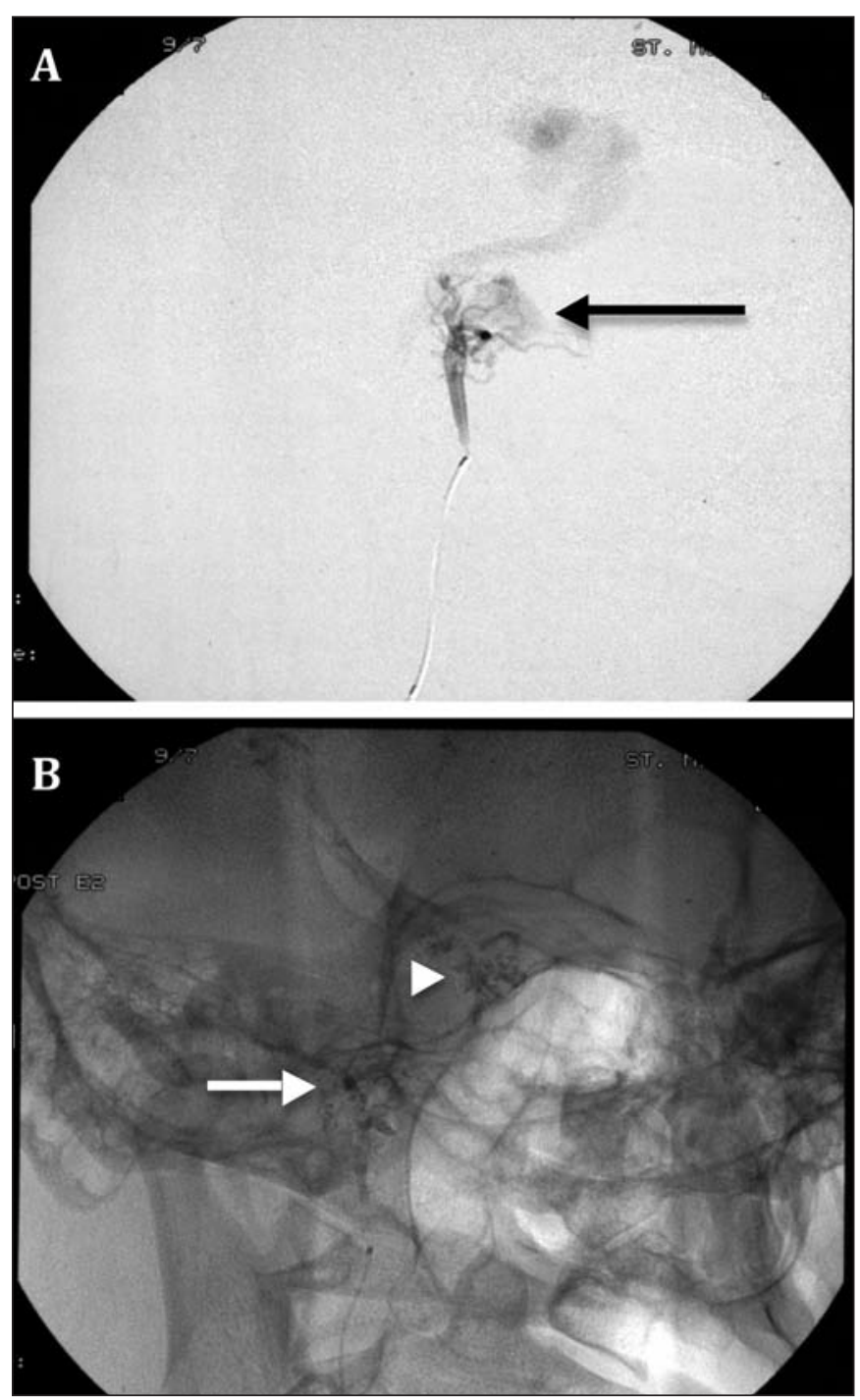

Figure 3: (A) Injection of the right ascending pharyngeal artery demonstrates nidus (arrow) at the hypoglossal canal with multiple additional feeders. (B) n-butyl-cyanoacrylate cast within the arterial $D A V F$ feeders with venous penetration of embolic material into the IPS (arrow) and cavernous sinus (arrowhead).

such as a nerve sheath tumor. Caution should be exercised when interpreting an enhancing structure protruding form a skull base foramen by first correlating with lack of patient symptomatology and considering a nearby DAVF or impaired internal jugular venous return with prominent veins as potential differential diagnostic possibilities. To aid in correct diagnosis, alternative imaging planes and flow-sensitive sequences can be used. ${ }^{14}$

Chemosis and proptosis are symptoms usually associated with retrograde venous drainage from arteriovenous fistulas in the region of the cavernous sinus [either DAVF or carotidcavernous fistula $(\mathrm{CCF})]$. In addition, it has been reported that clinical symptoms associated with marginal sinus arteriovenous 
fistulas can mimic those of $\mathrm{CCF}^{15}$ Although rare, dural fistulae in the region of the hypoglossal canal can present with ocular symptoms very similar to arteriovenous fistulae of the cavernous sinus region, and probably should be included in the differential diagnosis. Despite the limited number of cases available in the literature ocular symptoms appear to be a relatively common clinical expression of these lesions. ${ }^{16-18}$ Venous and arterial anatomy of the region of the hypoglossal canal and its connections with ocular vessels is complex and a clear understanding is essential before treatment can be planned. The vascular supply to the dura in the hypoglossal canal region includes distal branches of the ascending pharyngeal (AP) and occipital arteries. The AP artery consists of two major trunks, the pharyngeal trunk and the neuromeningeal trunk. From the neuromeningeal trunk arises the hypoglossal branch, jugular branch, internal auditory branch and clival branches, the most important being the former two. The hypoglossal branch traverses the hypoglossal canal to supply the posterior fossa meninges and the vasa nervorum of cranial nerve XII with a posterior descending branch contributing to the odontoid arch system. The jugular branch extends to the posterior fossa to the jugular foramen and supplies the vasa nervorum of cranial nerves IX, X and XI. Several small branches leave the jugular foramen to supply the meninges of the internal auditory canal, the dura of the IPS and possibly the vasa nervorum of cranial nerve VI. The occipital artery is a branch of the external carotid artery and infrequently from the internal carotid artery. It courses obliquely posteriorly to the medial surface of the mastoid process. As it penetrates the skull though the emissary foramen it bifurcates into one branch that courses antero-laterally and joins the meningeal branches of the ascending pharyngeal artery and another branch that courses postero-superiorly to join the branches of the posterior meningeal artery. The venous plexus within the hypoglossal canal is referred to as the anterior condylar vein. It originates at the junction of the jugular bulb and IPS and transverses the hypoglossal canal to join the IPS. The venous network at the skull base is complex, including the marginal sinus, posterior condylar emissary vein, and the vertebral and basilar venous plexuses. This network provides the anatomical basis for the communication with the cavernous sinus and orbital veins if retrograde flow caused by a fistula is present. $^{18-21}$

Symptoms of cranial DAVFs are a consequence of the pattern of venous drainage. Fistulae with cortical venous reflux, more common in the tentorium and transverse-sigmoid sinus, have a high risk of hemorrhage, while ocular symptoms such as proptosis, chemosis, diploplia and increased intraocular pressure are usually seen in DAVFs of the region of the cavernous sinus..$^{3,4,22}$ One would expect that lesions located in the occipital bone, including the hypoglossal canal region, present themselves with symptoms such as bruit or, in the case of cortical venous reflux, hemorrhage. As our series and a review of the available literature of these rare lesions demonstrate, ${ }^{16,17}$ DAVF of the hypoglossal canal should be included in the differential diagnosis of ocular symptoms, despite the considerable distance between the hypoglossal canal and the orbital veins. As is the case with CCF, meticulous ophthalmologic care must be provided with serial visual acuity and intraocular pressure measurements. Any change, including improvement in the ophthalmologic symptoms, may signify changes in the fistula and requires prompt investigation. Considering the complexity of open surgical approaches to the region, with potential morbidity and significant blood loss, ${ }^{19,21,23}$ treatment should always include careful evaluation of endovascular options. Different endovascular approaches are described and treatment should be tailored to specific characteristics of each case, since size and accessibility of vascular supply and drainage may vary from case to case. Digital subtraction angiography (DSA) is a standard examination for diagnosing the DAVF and detailed examination of its arterial supply and venous drainage pathways. The location and intraosseous extent of the lesion can be effectively studied using computed tomography and magnetic resonance angiography. Endovascular treatment options include: transvenous embolization from ipsilateral jugular vein or from the contralateral jugular vein across the IPS to the ipsilateral fistula, embolization via superior ophthalmic vein to hypoglossal canal region and multiple selective trans-arterial embolizations. Understanding of the complex venous anatomy at the skull base and its possible connections is essential in planning the endovascular approach. Trans-arterial embolization with liquid embolic agents, n-butyl-cyanoacrylate (NBCA) or OnyxR (EV3), is known to carry significant risk including lower cranial nerve palsies and neurointerventionalists must have a comprehensive understanding of the local anatomy and associated anastomoses to minimize these risks. A complete angiographic study, including a careful evaluation of the brain venous drainage, is mandatory. When feasible, a transvenous approach through the jugular vein is the preferred treatment as access is relatively simple and transvenous embolization has a higher likelihood of achieving complete occlusion than transarterial embolization. When access from the jugular system is not feasible, either from the ipsilateral side or from the contralateral side across the IPS, a transvenous approach through a superior ophthalmic vein cut-down, as described for CCF, can be effective. While transvenous embolization is preferable, transarterial embolization can also be effective in selected cases, either as a curative treatment or in order to reduce flow through the fistula, palliating symptoms or as an adjuvant to other approaches. Superselective injection of embolic material in the occipital and ascending pharyngeal artery should be performed bearing in mind that connections with the vertebral artery are common.

Dural fistulae located in the hypoglossal canal region are rare. Despite the relative distance and apparent disconnection between the eyes and the hypoglossal canal, ocular symptoms are the presenting symptom in a significant percentage of patients who may present with cranial nerve neuropathy and/or symptoms of increased intraocular venous pressure. The treatment of these lesions can be challenging. Endovascular treatment is a very attractive option and should be carefully planned, taking into account arterial supply and venous drainage with particular attention to the complex anatomy with variable arterial supply and venous drainage patterns. Each patient in our series was managed using a different endovascular approach highlighting the heterogeneous nature of these rare lesions. 


\section{REFERENCES}

1. Borden JA, Wu JK, Shucart WA. A proposed classification for spinal and cranial dural arteriovenous fistulous malformations and implications for treatment. J Neurosurg. 1995;82:166-79.

2. Satomi J, van Dijk JM, ter Brugge KG, Willinsky RA, Wallace MC. Benign cranial dural arteriovenous fistulas: Outcome of conservative management based on the natural history of the lesion. J Neurosurg. 2002;97:767-70.

3. van Dijk JM, ter Brugge KG. Dural arteriovenous fistulas. J Neurosurg. 2002;97:1486; author reply 1486-7.

4. van Dijk JM, ter Brugge KG, Willinsky RA, Wallace MC. Clinical course of cranial dural arteriovenous fistulas with long-term persistent cortical venous reflux. Stroke. 2002;33:1233-6.

5. Willinsky R, Goyal M, ter Brugge K, Montanera W. Tortuous, engorged pial veins in intracranial dural arteriovenous fistulas: correlations with presentation, location, and MR findings in 122 patients. AJNR Am J Neuroradiol. 1999;20:1031-6.

6. Safavi-Abbasi S, Di Rocco F, Nakaji P, Feigl GC, Gharabaghi A, Samii M, et al. A Thrombophilia due to factor $v$ and factor ii mutations and formation of a dural arteriovenous fistula: case report and review of a rare entity. Skull Base. 2008;18:135-43.

7. van Dijk JM, ter Brugge KG, Van der Meer FJ, Wallace MC, Rosendaal FR. Thrombophilic factors and the formation of dural arteriovenous fistulas. J Neurosurg. 2007; 107:56-9.

8. Flannery T, Tan MH, Flynn P, Choudhari KA. Delayed post-surgical development of dural arteriovenous fistula after cervical meningocele repair. Neurol India. 2003;51:390-1.

9. Yassari R, Jahromi B, Macdonald R. Dural arteriovenous fistula after craniotomy for pilocytic astrocytoma in a patient with protein s deficiency. Surg Neurol. 2002;58:59-64; discussion 64.

10. Singh V, Meyers PM, Halbach VH, Gress DR, Higashida RT, Dowd $\mathrm{CF}$, et al. Dural arteriovenous fistula associated with prothrombin gene mutation. J Neuroimaging. 2001;11:319-21.

11. Witt O, Pereira PL, Tillmann W. Severe cerebral venous sinus thrombosis and dural arteriovenous fistula in an infant with protein s deficiency. Childs Nerv Syst. 1999;15:128-30.

12. Uranishi R, Nakase H, Sakaki T. Expression of angiogenic growth factors in dural arteriovenous fistula. J Neurosurg. 1999;91: 781-6.
13. Feldman RA, Hieshima G, Giannotta SL, Gade GF. Traumatic dural arteriovenous fistula supplied by scalp, meningeal, and cortical arteries: case report. Neurosurgery. 1980;6:670-4.

14. Stuckey SL. Dilated venous plexus of the hypoglossal canal mimicking disease. AJNR Am J Neuroradiol. 1999;20:157-8.

15. Turner RD, Gonugunta V, Kelly ME, Masaryk TJ, Fiorella DJ. Marginal sinus arteriovenous fistulas mimicking carotid cavernous fistulas: diagnostic and therapeutic considerations. AJNR Am J Neuroradiol. 2007;28:1915-8.

16. Tanoue S, Goto K, Oota S. Endovascular treatment for dural arteriovenous fistula of the anterior condylar vein with unusual venous drainage: report of two cases. AJNR Am J Neuroradiol. 2005;26:1955-9.

17. Kiyosue H, Tanoue S, Okahara M, Mori M, Mori H. Ocular symptoms associated with a dural arteriovenous fistula involving the hypoglossal canal: selective transvenous coil embolization. Case report. J Neurosurg. 2001;94:630-2.

18. Ernst R, Bulas R, Tomsick T, van Loveren H, Aziz KA. Three cases of dural arteriovenous fistula of the anterior condylar vein within the hypoglossal canal. AJNR Am J Neuroradiol. 1999;20: 2016-20.

19. Wen HT, Rhoton AL, Jr., Katsuta T, de Oliveira E. Microsurgical anatomy of the transcondylar, supracondylar, and paracondylar extensions of the far-lateral approach. J Neurosurg. 1997;87: 555-85.

20. San Millan Ruiz D, Gailloud P, Rufenacht DA, Delavelle J, Henry F, Fasel JH. The craniocervical venous system in relation to cerebral venous drainage. AJNR Am J Neuroradiol. 2002;23: 1500-8.

21. Katsuta T, Rhoton AL, Jr., Matsushima T. The jugular foramen: microsurgical anatomy and operative approaches. Neurosurgery. 1997;41:149-201; discussion 201-2.

22. Lasjaunias P, Berenstein A, ter Brugge, KG. Surgical neuroangiography: 1 clinical vascular anatomy and variations. 2nd ed. Berlin: Springer-Verlag; 2001.

23. Tirakotai W, Benes L, Kappus C, Sure U, Farhoud A, Bien S, et al. Surgical management of dural arteriovenous fistulas with transosseous arterial feeders involving the jugular bulb. Neurosurg Rev. 2007;30:40-8; discussion 48-9. 